\title{
Linearizing Nonlinear Optics
}

\author{
Bruno E. Schmidt ${ }^{1}$, Philippe Lassonde ${ }^{2}$, Guilmot Ernotte $^{2}$, Matteo Clerici $^{3}$, Roberto \\ Morandotti $^{2}$, Heide Ibrahim $^{2}$ and François Légaré \\ ${ }^{I}$ few-cycle Inc., 2890 Rue de Beaurivage, Montreal, H1L 5W5, Qc, Canada \\ ${ }^{2} I N R S-E M T, 1650$ Blvd. Lionel Boulet, Varennes, J3X 1S2, Qc, Canada \\ ${ }^{3}$ University of Glasgow, School of Engineering, G12 8QQ, Glasgow, UK
}

\begin{abstract}
Fourier nonlinear optics merges the simplicity of linear optics with the power of nonlinear optics to achieve a decoupling of frequencies, amplitudes and phases in nonlinear processes - enabling first deep UV shaping at $207 \mathrm{~nm}$.
\end{abstract}

\section{Introduction}

Unlike in linear optics, broadband fs laser pulses can interact nonlinearly in a medium which couples their amplitudes phases in a convolution process.

Here, we describe a new regime where nonlinear interactions can occur without mixing of different frequencies [1]. We demonstrate how frequency domain nonlinear optics (FNO) overcomes the shortcomings arising from the convolution in conventional time-domain nonlinear optics (TNO). We generate light fields with previously inaccessible properties by evoking the uncontrolled coupling of amplitudes and phases. We show that arbitrary phase functions are transferred linearly to the second harmonic frequencies while the output spectrum is exactly equal to the square of the input. Our current work provides a generalized description of FNO that also covers the recently introduced Frequency domain Optical Parametric Amplification (FOPA) [2].

\section{Experimental results}

In TNO (Fig. 1b), all frequencies interact simultaneously in a single focus and the second harmonic spectrum (blue curve) shows a smooth function without the initial central hole. Aa result of the convolution. 
In contrast, the key aspect of FNO, shown in Fig. 1c, is the intrinsic ability to achieve a very narrow bandwidth for the nonlinear light-matter interaction even though the input pulse contains a broad bandwidth. Experimentally, this is realized by aid of a $4 \mathrm{f}$ configuration [3]. The optical FT provides a reduced frequency content in each focal spot of the frequency plane where the nonlinear interaction takes place. All these independent picosecond interactions are coherently recombined at the second grating $\mathrm{G} 2$ denoting a second Fourier transformation back to the time domain. To compare TNO and FNO, we prepare an input spectrum (Fig. 1a) corresponding to transform limited $33 \mathrm{fs}$ pulses at $800 \mathrm{~nm}$ wavelength but exhibiting a spectral hole in the center. This input is frequency doubled either via TNO as is shown in Fig. $1 \mathrm{~b}$ or via FNO, see Fig. 1c. In both cases, we use a $150 \mu \mathrm{m}$ thin BBO as the nonlinear crystal (NC) to minimize the role of phase-mismatch. In TNO (Fig. 1b), all frequencies interact simultaneously in a single focus and the experimental SH spectrum (blue curve) shows a smooth function without the initial central hole, a result of the convolution.

$$
\text { For time-domain interactions: } \quad E_{T}^{S H}(\omega) \propto E_{T}(\omega) * E_{T}(\omega)
$$

In FNO (Fig. 1c), the frequencies of an incident pulse disperse after the first grating G1 and their different propagation angles become parallel after the first lens L1. The lens again focuses each plane wave into a small focal spot next to its neighboring frequency. All frequency components, located side by side now form the frequency plane at one focal length

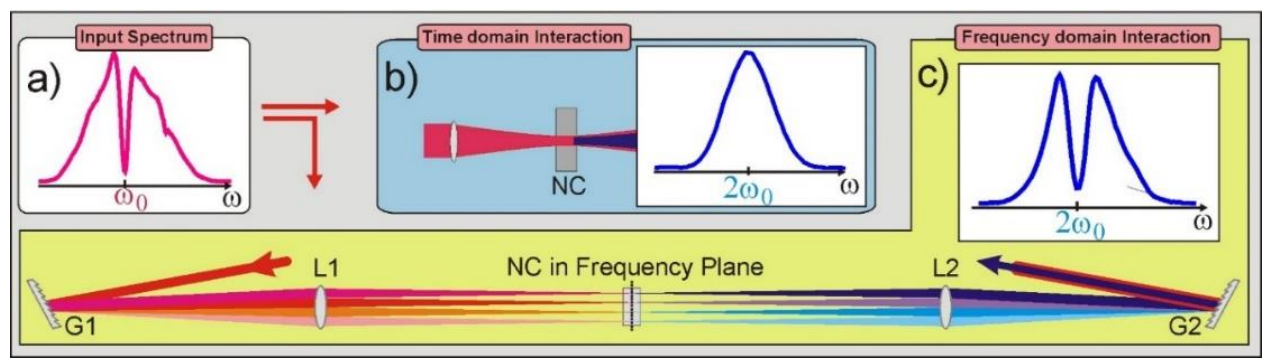

Fig. 1: A shaped input spectrum (a) is frequency doubled either in (b) conventional time domain nonlinear optics (TNO) or (c) via Frequency domain nonlinear optics (FNO). The convolution of different frequencies in TNO (b) leads to a smoothening of the input function while in FNO the shape is preserved since the mutual frequency crosstalk is turned off. This decoupling permits a transfer of arbitrary phase functions. NC: nonlinear crystal.

behind the lens. This setup performs a continuous optical Fourier transformation from the time to the frequency domain. A symmetric setup subsequent to the frequency plane performs a second Fourier transformation back to the time domain. The experimentally obtained $\mathrm{SH}$ spectrum at the output of the $4 \mathrm{f}$ setup (blue curve in fig. 1c) exhibits the same shape as the input spectrum.

We developed an analytical theory to describe FNO [1], and in the case of second harmonic generation, the output field is given by the equation

$$
\begin{aligned}
& \text { For frequency domain interaction: } \\
& \qquad E_{F}^{S H}(2 \omega) \propto E_{F}(\omega) \cdot E_{F}(\omega)=|E(\omega)|^{2} e^{i 2 \phi(\omega)}
\end{aligned}
$$

The convolution of the E-fields in Eq. (1) is now changed to a simple multiplication in Eq. (2). This crucial difference is the foundation of FNO since it contains: (i) direct amplitude transfer, (ii) linear phase transfer and (iii) the decoupling of amplitudes and phases.

As apowerful application example for arbitrairy phase transfer, we utilize FNO for first time deep UV pulse shaping at the 4th harmonic of the TiSa laser. We stress that this phase shaping (and straightforwardly amplitude shaping) is carried out with a conventional shaper 
prior to the TiSa amplifier. To reach the deep UV range we imaged the first Fourier plane generating the $\mathrm{SH}$ onto a subsequent one to double once more the frequency with a BBO crystal. The results of transformlimited output at 207nm shown in Fig. 2a) and a pulse train obtained through pure sinusoidal phase shaping at $800 \mathrm{~nm}$ in Fig. $2 \mathrm{~b}$ underline the new oportunities ariesing through FNO.

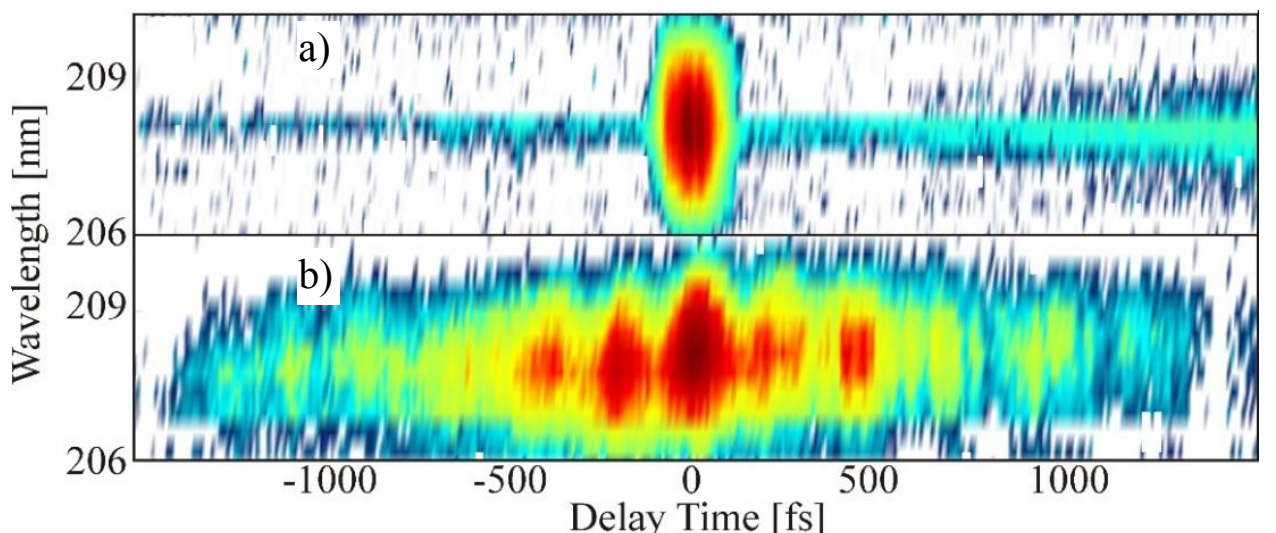

Fig. 2: First time deep UV phase shaping and transient grating frequency resloved optical gating (TG-FROG). Transform limited UV pulses in (a) are trasnfered into a pulse train (b) through sinusoidal phase shaping at the fundamental priorr to the TiSa amplifier and subsequent frequency quadripling in accordig to a Fourier nonliner optics scheme.

\section{Conclusion}

We explain and demonstrate how frequency domain nonlinear optics enables the linear transfer of arbitrary phase functions as well as the decoupling of amplitudes and phases and why this is not possible in time domain interaction. A powerful application is deep UV shaping at $207 \mathrm{~nm}$.

\section{References}

[1] Schmidt et al., "Decoupling Frequencies, Amplitudes and Phases in Nonlinear Optics“, Scientific reports 7, 7861 (2017).

[2] Schmidt et al., "Frequency domain optical parametric amplification," Nature Com., 5, 3643 (2014).

[3] Froehly et al., "Progress in Optics," 20, 63-153 (Elsevier, 1983). 